\title{
Continuous positive airway pressure and noninvasive ventilation in prehospital treatment of patients with acute respiratory failure: a systematic review of controlled studies
}

\author{
Skule A Bakke ${ }^{1 *}$, Morten T Botker ${ }^{2}$, Ingunn S Riddervold ${ }^{2}$, Hans Kirkegaard ${ }^{3}$ and Erika F Christensen ${ }^{2}$
}

\begin{abstract}
Continuous positive airway pressure (CPAP) and noninvasive ventilation (NIV) are frequently used inhospital for treating respiratory failure, especially in treatment of acute cardiogenic pulmonary edema and exacerbation of chronic obstructive pulmonary disease. Early initiation of treatment is important for success and introduction already in the prehospital setting may be beneficial. Our goal was to assess the evidence for an effect of prehospital CPAP or NIV as a supplement to standard medical treatment alone on the following outcome measures; mortality, hospital length of stay, intensive care unit length of stay, and intubation rate. We undertook a systematic review based on a search in the three databases: PubMed, EMBASE, and Cochrane. We included 12 studies in our review, but only four of these were of acceptable size and quality to conclude on our endpoints of interest. All four studies examine prehospital CPAP. Of these, only one small, randomized controlled trial shows a reduced mortality rate and a reduced intubation rate with supplemental CPAP. The other three studies have neutral findings, but in two of these a trend toward lower intubation rate is found. The effect of supplemental NIV has only been evaluated in smaller studies with insufficient power to conclude on our endpoints. None of these studies have shown an effect on neither mortality nor intubation rate, but two small, randomized controlled trials show a reduction in intensive care unit length of stay and a trend toward lower intubation rate. The risk of both type two errors and publication bias is evident, and the findings are not consistent enough to make solid conclusion on supplemental prehospital NIV. Large, randomized controlled trials regarding the effect of NIV and CPAP as supplement to standard medical treatment alone, in the prehospital setting, are needed.
\end{abstract}

Keywords: Prehospital, Continuous positive airway pressure, Noninvasive ventilation, Respiratory failure, Acute pulmonary edema, Chronic obstructive pulmonary disease, Mortality, Hospital length of stay, Intensive care unit length of stay, Intubation rate

\section{Introduction}

Dyspnea is a frequent symptom among patients in the prehospital setting [1]. Common causes of nontraumatic dyspnea are congestive heart failure, pneumonia, chronic obstructive pulmonary disease, and asthma [1]. The application of advanced airway management and alternative devices in the prehospital setting has recently been

\footnotetext{
* Correspondence: skulebakke@hotmail.com

'Department of Anesthesiology, Hospital of Southern Jutland, Southern Jutland, Denmark

Full list of author information is available at the end of the article
}

defined as one of the top priority research questions in physician-provided prehospital critical care [2].

Continuous positive airway pressure (CPAP) and noninvasive ventilation (NIV) are often used in intensive care units for treating respiratory failure caused by acute cardiogenic pulmonary edema (ACPE) and acute exacerbation of chronic obstructive pulmonary disease (COPD). CPAP-systems apply positive airway pressure with only minimal differences in the pressure applied during inspiration and expiration [3]. The term NIV covers different forms of noninvasive positive pressure ventilation, which in contrast to CPAP can also ad extra

\section{() Biomed Central}

(c) 2014 Bakke et al.; licensee BioMed Central Ltd. This is an Open Access article distributed under the terms of the Creative Commons Attribution License (http://creativecommons.org/licenses/by/4.0), which permits unrestricted use, distribution, and reproduction in any medium, provided the original work is properly credited. The Creative Commons Public Domain Dedication waiver (http://creativecommons.org/publicdomain/zero/1.0/) applies to the data made available in this article, unless otherwise stated. 
inspiratory support driven by a ventilator, thereby giving positive pressure ventilation $[3,4]$. Standard medical treatment given for acute respiratory failure is diverse, depending on assumed cause and type of emergency medical staffing. It ranges from simple supplemental oxygen therapy to nitrates, diuretics, opioids, inhaled bronchodilators, and inotropic infusions. The worst cases can result in endotracheal intubation.

Recent Cochrane reviews show lower mortality and reduced intubation rate with the use of inhospital supplemental CPAP and NIV, compared to standard medical treatment alone, in patients with ACPE and exacerbations of COPD [5,6]. Lower intubation rates decrease the risk of complications related to endotracheal intubation and invasive ventilation, especially pulmonary infections [7-9].

Prehospital intubation is associated with high successrates in physician-staffed services [10]. However, aspiration of gastric contents during intubation is reported more frequent in the prehospital setting than in the emergency department [11]. One study reports complications in $14 \%$ of prehospital advanced airway managements [12]. Especially vomiting, hypotension, and hypoxia do occur, but only a minor proportion of the patients in this study would have been suitable for CPAP/ NIV as only $21 \%$ were intubated because of hypoxia. More than half of the patients had cardiac arrest. A prerequisite for successful noninvasive treatment is early initiation of CPAP or NIV $[13,14]$. Thus it is reasonable to believe that many patients would benefit from earlier initiation of noninvasive treatment, in the prehospital setting, to avoid intubation and improve patient outcome.

The objective of this systematic review of controlled studies was to examine, whether CPAP or NIV initiated in the prehospital setting reduce mortality, abbreviate hospital length of stay (H-LOS), abbreviate intensive care unit length of stay (ICU-LOS), or lower intubation rate when used as a supplement to standard medical treatment alone.

\section{Review}

\section{Methods}

Published studies relevant for this review were identified by a search in the databases PubMed, EMBASE, and Cochrane on April $4^{\text {th }} 2013$ and updated January $19^{\text {th }}$ 2014. Our inclusion criteria were: Controlled studies examining the effect of supplemental prehospital CPAP or NIV, compared to standard medical treatment alone, in adult patients with acute respiratory failure of any cause. In PubMed the following search string was used: "Continuous Positive Airway Pressure" [Mesh] OR "Noninvasive Ventilation" [Mesh] OR non invasive ventilation) AND ("Respiratory Insufficiency" [Mesh] OR "Pulmonary disease, Chronic obstructive" [Mesh] OR "Heart Failure"[Mesh] OR "Pulmonary Edema" [Mesh] OR "Asthma" [Mesh]) AND ("Emergency medical services"
[Mesh] OR prehospital OR pre-hospital OR out of hospital).

We systematically excluded studies that did not meet the inclusion criteria in a hierarchical manner according to the following exclusion criteria:

1. Studies not regarding CPAP or NIV

2. Not prehospital setting

3. Not acute respiratory failure of any cause

4. Not a clinical trial

5. Not a controlled design comparing supplemental CPAP or NIV to standard medical treatment alone

6. Not adult patients ( $\geq 18$ years)

7. Abstract only

First the title of a study, as it appeared from the search pages in the respective databases, was read and searched for the exclusion criteria described above. If a study could not be excluded based on its title, the abstract was read. Based on the abstract, we excluded studies that did not meet the inclusion criteria in the same hierarchical manner. If exclusion could not be done based on the abstract, the entire article was read. By this selection process, studies with inhome use of noninvasive ventilation for chronic pulmonary disorders, CPAP or NIV during intrahospital transport, expert opinions, editorials, reports, and case series were excluded. Duplications and conference abstracts were removed. Two reviewers independently carried out the searches, and discrepancies regarding exclusion were solved by consensus. Subsequently, a hand-search through references in the included studies, relevant reviews, and the "related citations" feature on PubMed was performed. Two reviewers independently extracted study details from the included articles, searching for our endpoints of interest: mortality, H-LOS, ICU-LOS, and intubation rate. Discrepancies regarding data extraction were solved by consensus. The included studies were independently evaluated by two reviewers according to the Scottish Intercollegiate Guidelines Network 50 (SIGN 50) checklist for randomized and/ or controlled trials [15]. SIGN implements the Grading of Recommendations Assessment, Development, and Evaluation (GRADE) Working Groups approach within its methodology. The SIGN 50 checklists section one shows quality of evidence rated in one of four categories (ranging from "well covered" to "not addressed"). Section two starts by rating the methodological quality of the study, based on answers in section one and using the following coding system: High quality $(++)$ : The majority of criteria are met. There is little risk of bias. Results are unlikely to be changed by further research. Acceptable quality (+): Most of the criteria are met. There could be some flaws in the study with an associated risk of bias. Conclusions may be changed by further research. Low quality (-): Either most criteria are not met, or there are significant flaws relating 
to key aspects of study design. We were aware that included studies would be heterogeneous, but used the same checklist to assess all types of controlled studies in order to improve the systematic approach and critical evaluation.

To minimize bias, two reviewers evaluated each study independently. Differences in assessment were discussed and discrepancies solved by consensus with a third reviewer. We have presented the results from all studies, but our conclusion is based only on studies rated + or ++ .

\section{Results}

We located 196 published studies searching PubMed, 290 studies searching EMBASE, and 228 studies searching Cochrane, yielding a total of 714 studies. Of these, 700 studies were excluded based on titles or abstracts. One study was found by hand-search [16] and, a total of 15 full-text articles, were read (Figure 1). We subsequently excluded one study that examines CPAP alone compared to CPAP and standard medical treatment [17]. Another study examines CPAP and medical treatment given at different time intervals, and not supplemental CPAP compared to standard medical treatment alone. It was therefore excluded [18]. Finally, one study was excluded because it is a cost-benefit analysis and not a clinical trial [19]. One study does not report if standard medical treatment was used in the intervention group and an email was sent to the corresponding author for clarification, but there was no reply. Based on our evaluation of the methods section, both intervention and control groups received medical treatment and the study was thus included for analysis [20]. Another study does not state a primary endpoint, but reports on endpoints of interest for our review and was therefore included [21]. Thus we included 12 studies for final analysis [16,20-30]. Of the included studies, eight studies examine CPAP as intervention $[16,22-25,27,28,30]$ and four studies examine NIV as intervention [20,21,26,29].

Overview of study design and main findings of the studies can be found in Table 1. According to our evaluation of clarity and quality of the included studies, by use of the SIGN 50 checklist, eight studies either lack power to conclude on our outcomes of interest or entails high risk of bias [20-22,24-27,29]. Four studies have acceptable power and risk of bias $[16,23,28,30]$. The clarity and quality of the studies, according to the SIGN 50 checklist, is shown in Table 2.

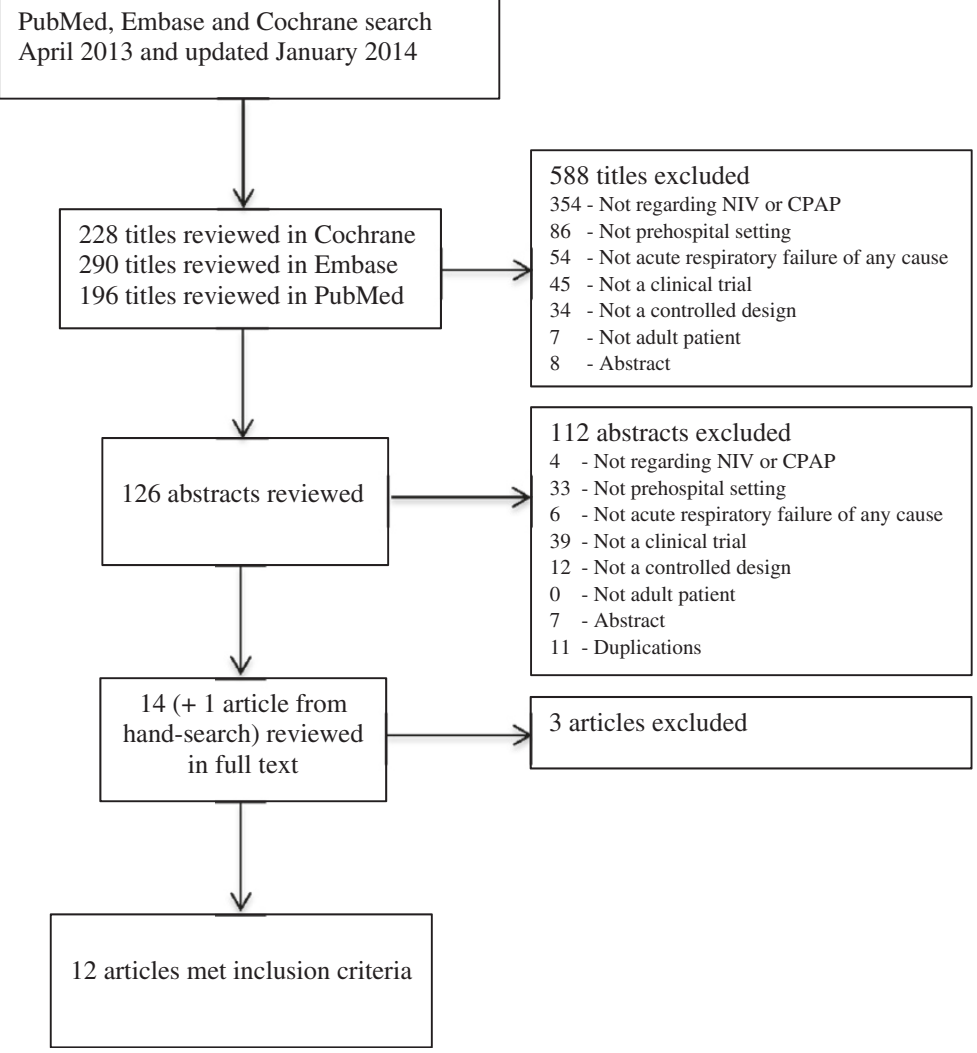

Figure 1 Search flow diagram. 
Table 1 Characteristics of included studies comparing standard medical treatment with supplementary CPAP or NIV

\begin{tabular}{|c|c|c|c|c|c|c|c|c|c|}
\hline Study & $\begin{array}{l}\text { Study } \\
\text { Country }\end{array}$ & Intervention & Study design & Number of patients $(\mathrm{N})$ & $\begin{array}{l}\text { Crude } \\
\text { mortality } \\
\text { rates }\end{array}$ & $\begin{array}{l}\text { Type of } \\
\text { patients }\end{array}$ & $\begin{array}{l}\text { Primary } \\
\text { outcome } \\
\text { secondary } \\
\text { outcome(s) }\end{array}$ & $\begin{array}{l}\text { Primary } \\
\text { result } \\
\text { secondary } \\
\text { result(s) }\end{array}$ & $\begin{array}{l}\text { Primary } \\
\text { review } \\
\text { outcomes }\end{array}$ \\
\hline \multirow[t]{4}{*}{ Cheskes et al. [16] } & Canada & CPAP & $\begin{array}{l}\text { Observational, } \\
\text { before-and-after }\end{array}$ & 214 Interventions & $17 / 214$ & $\begin{array}{l}\text { ARF of any } \\
\text { cause }\end{array}$ & $\begin{array}{l}\text { Mortality, } \\
\text { in-hospital }\end{array}$ & $\begin{array}{l}7.9 \% \text { vs. } 7.5 \% \\
(p=0.85)\end{array}$ & $\begin{array}{l}\text { Mortality: } \\
\rightarrow\end{array}$ \\
\hline & & & & 228 Controls & $17 / 228$ & & & & $\begin{array}{l}\text { H-LOS: } \\
\text { N/R }\end{array}$ \\
\hline & & & & & & & & & $\begin{array}{l}\text { ICU-LOS: } \\
\text { N/R }\end{array}$ \\
\hline & & & & & & & intubation rate & $\begin{array}{l}14.5 \% \text { vs. } \\
12.7 \% \\
(p=0.59)\end{array}$ & $\mathrm{IR}: \rightarrow$ \\
\hline \multirow[t]{4}{*}{$\begin{array}{l}\text { Dib et al. } \\
\text { [27] }\end{array}$} & USA & CPAP & $\begin{array}{l}\text { Retrospective, } \\
\text { controlled }\end{array}$ & 149 Interventions & $N / R$ & $\begin{array}{l}\text { Presumed } \\
\text { ACPE }\end{array}$ & $\begin{array}{l}\text { Prehospital treatment } \\
\text { times }\end{array}$ & $\begin{array}{l}30 \text { vs. } 31 \mathrm{~min} \\
(p>0.01)\end{array}$ & $\begin{array}{l}\text { Mortality: } \\
\text { N/R }\end{array}$ \\
\hline & & & & & & & & & H-LOS: N/R \\
\hline & & & & & & & & & ICU-LOS: N/R \\
\hline & & & & 238 Controls & $N / R$ & & $\begin{array}{l}\text { clinical variables } \\
\text { intubation rate }\end{array}$ & $\begin{array}{l}\text { improvement } \\
(p ' s<0.01) \\
2.6 \% \text { vs. } 4.6 \% \\
(p<0.01)\end{array}$ & IR: $\downarrow$ \\
\hline \multirow[t]{3}{*}{ Ducros et al. [30] } & France & CPAP & $\begin{array}{l}\text { Randomized, } \\
\text { controlled } \\
\text { multicentre }\end{array}$ & 107 Interventions & $8 / 107$ & $\begin{array}{l}\text { Presumed } \\
\text { ACPE }\end{array}$ & $\begin{array}{l}\text { Combined criteria } \\
\text { (successful) }\end{array}$ & $\begin{array}{l}\text { Odds ratio } 2.1 \\
(1.2-4.0)\end{array}$ & $\begin{array}{l}\text { Mortality: } \\
\rightarrow \\
\text { H-LOS: N/R }\end{array}$ \\
\hline & & & & & & & & & ICU-LOS: $\rightarrow$ \\
\hline & & & & 100 Controls & $9 / 100$ & & $\begin{array}{l}\text { Mortality, } 48 \text { hours } \\
\text { mortality, in hospital } \\
\text { Intensive care } \\
\text { unit length-of-stay }\end{array}$ & $\begin{array}{l}\text { odds ratio } 1.4 \\
\text { (0.4-5.2) } \\
\text { odds ratio } 0.9 \\
\text { (0.4-2.5) } \\
2 \text { vs. } 2 \text { days } \\
(p=0.67)\end{array}$ & $\mathrm{IR}: \rightarrow$ \\
\hline \multirow[t]{4}{*}{ Frontin et al. [28] } & France & CPAP & $\begin{array}{l}\text { Randomized, } \\
\text { controlled }\end{array}$ & 62 Interventions & $6 / 60$ & $\begin{array}{l}\text { Presumed } \\
\text { ACPE }\end{array}$ & Treatment success & $\begin{array}{l}\text { Odds ratio } 1.19 \\
(0.56-2.53)\end{array}$ & $\begin{array}{l}\text { Mortality: } \\
\rightarrow\end{array}$ \\
\hline & & & & & & & & & H-LOS: $\rightarrow$ \\
\hline & & & & & & & & & ICU-LOS: $\rightarrow$ \\
\hline & & & & 62 Controls & $7 / 62$ & & $\begin{array}{l}\text { Intubation rate } \\
\text { hospital } \\
\text { length-of-stay } \\
\text { Intensive } \\
\text { care unit } \\
\text { length-of-stay } \\
\text { mortality, } \\
30 \text { days }\end{array}$ & $\begin{array}{l}\text { odds ratio } 1,47 \\
\text { (0.23-9.23) } \\
6 \text { vs. } 6 \text { days } \\
(p=0,5) \\
8,2 \text { vs. } 8 \text { hours } \\
(p=0,27) \\
\text { odds ratio } \\
1.14(0.36-3.65)\end{array}$ & $\mathrm{IR}: \rightarrow$ \\
\hline
\end{tabular}


Table 1 Characteristics of included studies comparing standard medical treatment with supplementary CPAP or NIV (Continued)

\begin{tabular}{|c|c|c|c|c|c|c|c|c|c|}
\hline \multirow[t]{4}{*}{ Gardtman et al. [22] } & \multirow[t]{4}{*}{ Sweden } & \multirow[t]{4}{*}{ CPAP } & \multirow[t]{4}{*}{$\begin{array}{l}\text { Observational, } \\
\text { before-and-after }\end{array}$} & 158 Interventions & $18 / 158$ & \multirow[t]{4}{*}{$\begin{array}{l}\text { Presumed } \\
\text { ACPE }\end{array}$} & $\begin{array}{l}\text { ACPE at } \\
\text { admission }\end{array}$ & $\begin{array}{l}76 \% \text { vs. } 93 \% \\
(p<0.0001)\end{array}$ & $\begin{array}{l}\text { Mortality: } \\
\rightarrow\end{array}$ \\
\hline & & & & \multirow[t]{3}{*}{158 Controls } & \multirow[t]{3}{*}{$18 / 158$} & & \multirow[t]{3}{*}{ mortality, 1 year } & \multirow{3}{*}{$\begin{array}{l}22 \% \text { vs. } 27 \% \\
(p=0.64)\end{array}$} & H-LOS: $\rightarrow$ \\
\hline & & & & & & & & & $\begin{array}{l}\text { ICU-LOS: } \\
\text { N/A }\end{array}$ \\
\hline & & & & & & & & & IR: N/A \\
\hline \multirow[t]{4}{*}{ Garuti et al. [25] } & \multirow[t]{4}{*}{ Italy } & \multirow[t]{4}{*}{ CPAP } & \multirow{4}{*}{$\begin{array}{l}\text { Prospective, } \\
\text { observational } \\
\text { with historical } \\
\text { control group }\end{array}$} & 35 Interventions & $1 / 35$ & \multirow[t]{4}{*}{$\begin{array}{l}\text { ARF of any } \\
\text { cause }\end{array}$} & Mortality, adjusted & $\begin{array}{l}\text { Odds ratio } 0.06 \\
(0.01-0.53)\end{array}$ & Mortality: $\downarrow$ \\
\hline & & & & \multirow[t]{3}{*}{125 Controls } & \multirow[t]{3}{*}{$30 / 125$} & & \multirow{3}{*}{$\begin{array}{l}\text { intubation } \\
\text { rate hospital } \\
\text { length-of-stay }\end{array}$} & \multirow{3}{*}{$\begin{array}{l}\text { no intubations } \\
12 \text { vs. 18.8 days } \\
(p<0.0001)\end{array}$} & H-LOS: $\downarrow$ \\
\hline & & & & & & & & & $\begin{array}{l}\text { ICU-LOS: } \\
\text { N/A }\end{array}$ \\
\hline & & & & & & & & & $\mathrm{IR}: \rightarrow$ \\
\hline \multirow[t]{4}{*}{ Hubble et al. [24] } & \multirow[t]{4}{*}{ USA } & \multirow[t]{4}{*}{ CPAP } & \multirow[t]{4}{*}{$\begin{array}{l}\text { Prospective, demographically } \\
\text { controlled }\end{array}$} & \multirow[t]{4}{*}{$\begin{array}{l}120 \text { Interventions } 95 \\
\text { Controls }\end{array}$} & $5.35 \%$ & \multirow[t]{4}{*}{$\begin{array}{l}\text { Presumed } \\
\text { ACPE }\end{array}$} & Intubation rate & $\begin{array}{l}\text { Odds ratio } \\
4.04(1.64-9.95)\end{array}$ & Mortality: $\downarrow$ \\
\hline & & & & & \multirow[t]{3}{*}{$23.15 \%$} & & \multirow{3}{*}{$\begin{array}{l}\text { mortality, in hospital } \\
\text { hospital length-of-stay }\end{array}$} & \multirow{3}{*}{$\begin{array}{l}\text { odds ratio } 7.48 \\
(1.96-28.54) \\
5.58 \text { vs. } 7.66 \text { days } \\
(p=0.755)\end{array}$} & H-LOS: $\rightarrow$ \\
\hline & & & & & & & & & $\begin{array}{l}\text { ICU-LOS: } \\
\text { N/A }\end{array}$ \\
\hline & & & & & & & & & IR: $\downarrow$ \\
\hline \multirow[t]{4}{*}{ Thompson et al. [23] } & \multirow[t]{4}{*}{ Canada } & \multirow[t]{4}{*}{ CPAP } & \multirow[t]{4}{*}{ Randomized, controlled } & 35 Interventions & $5 / 35$ & \multirow[t]{4}{*}{$\begin{array}{l}\text { ARF of any } \\
\text { cause }\end{array}$} & Intubation rate & $\begin{array}{l}\text { Odds ratio } \\
0.16(0.04-0.7)\end{array}$ & Mortality: $\downarrow$ \\
\hline & & & & 34 Controls & $12 / 34$ & & mortality, in hospital & $0.3(0.09-0.99)$ & H-LOS: $\rightarrow$ \\
\hline & & & & & & & Intensive care unit & 6.5 vs. 3 days & ICU-LOS: $\rightarrow$ \\
\hline & & & & & & & length-of-stay & & $\mathbb{R}: \downarrow$ \\
\hline Craven et al. [29] & USA & NIV & $\begin{array}{l}\text { Prospective, demographically } \\
\text { controlled }\end{array}$ & 37 Interventions & $6 / 37$ & $\begin{array}{l}\text { Presumed } \\
\text { ACPE }\end{array}$ & $\begin{array}{l}\text { Out of hospital } \\
\text { treatment time }\end{array}$ & $\begin{array}{l}31.4 \text { vs. } 31.2 \\
\min (p=0.931)\end{array}$ & Mortality: $\rightarrow$ \\
\hline & & & & 25 Controls & $2 / 24$ & & improvement in SpO2 & $13.71 \%$ vs. $6.99 \%$ & H-LOS: $\rightarrow$ \\
\hline & & & & & & & $\begin{array}{l}\text { nospital length-of-stay } \\
\text { mortality, in-hospital }\end{array}$ & $\begin{array}{l}(p=0.03 /) \\
6.34 \text { vs. } 7.63 \\
(p=0.48)\end{array}$ & $\begin{array}{l}\text { ICU-LOS: } \\
\text { N/R }\end{array}$ \\
\hline & & & & & & & & $\begin{array}{l}6 / 37 \text { vs. } 2 / 24 \\
(p=0.462)\end{array}$ & $\mathrm{IR}: \rightarrow$ \\
\hline Roessler et al. [20] & Germany & NIV & Randomized, controlled & 25 Interventions & $1 / 24$ & $\begin{array}{l}\text { ARF of any } \\
\text { cause }\end{array}$ & $\begin{array}{l}\text { Efficiency of } \\
\text { treatment }\end{array}$ & $\begin{array}{l}100 \% \text { vs. } 80 \% \\
(p=0.05)\end{array}$ & $\begin{array}{l}\text { Mortality: } \\
\rightarrow\end{array}$ \\
\hline & & & & 26 Controls & $2 / 25$ & & survival, 28 days & $96 \%$ vs. $92 \%$ & H-LOS: $\rightarrow$ \\
\hline & & & & & & & $\begin{array}{l}\text { intensive care unit } \\
\text { lenath-of-stay hospital }\end{array}$ & $\begin{array}{l}(p=1.0) \\
13.9 \text { vs. } 17.4 \text { days }\end{array}$ & ICU-LOS: $\downarrow$ \\
\hline & & & & & & & length-of-stay & $(p=0,5)$ & $\mathrm{IR}: \rightarrow$ \\
\hline & & & & & & & & $\begin{array}{l}(p=0.03) \\
1 \text { vs. } 6 \\
(p=0.66)\end{array}$ & \\
\hline
\end{tabular}


Table 1 Characteristics of included studies comparing standard medical treatment with supplementary CPAP or NIV (Continued)

\begin{tabular}{|c|c|c|c|c|c|c|c|c|c|}
\hline \multirow[t]{2}{*}{$\begin{array}{l}\text { Schmidbauer et al. } \\
\text { [21] }\end{array}$} & \multirow[t]{2}{*}{ Germany } & \multirow[t]{2}{*}{ NIV } & \multirow[t]{2}{*}{ Randomized, controlled } & 18 Interventions & $0 / 18$ & \multirow[t]{2}{*}{$\begin{array}{l}\text { Presumed } \\
\text { COPD }\end{array}$} & Dyspnea score & $\begin{array}{l}\text { Improvement } \\
(p<0.001)\end{array}$ & Mortality: - \\
\hline & & & & 18 Controls & $0 / 18$ & & $\begin{array}{l}\text { respiration rate } \\
\text { other clinical variables } \\
\text { Intensive care } \\
\text { unit length-of-stay }\end{array}$ & $\begin{array}{l}\text { improvement } \\
(p=0.001) \\
\text { No diff in } \\
\text { other clinical } \\
\text { variables } \\
59 \text { vs. } 185 \\
(p=0.02)\end{array}$ & $\begin{array}{l}\text { H-LOS: } \rightarrow \\
\text { ICU-LOS: } \downarrow \\
\text { IR: } \rightarrow\end{array}$ \\
\hline \multirow[t]{2}{*}{ Weitz et al. [26] } & \multirow[t]{2}{*}{ Germany } & \multirow[t]{2}{*}{ NIV } & \multirow[t]{2}{*}{ Randomized, controlled } & 10 Interventions & $1 / 10$ & \multirow[t]{2}{*}{$\begin{array}{l}\text { Presumed } \\
\text { ACPE }\end{array}$} & $\begin{array}{l}\text { SpO2 at hospital } \\
\text { admission }\end{array}$ & $\begin{array}{l}97.3 \% \text { vs. } 89.5 \% \\
(p=0.002)\end{array}$ & $\begin{array}{l}\text { Mortality: } \\
\rightarrow\end{array}$ \\
\hline & & & & 13 Controls & $1 / 13$ & & $\begin{array}{l}\text { clinical variables } \\
\text { Intensive care unit } \\
\text { length-of-stay } \\
\text { hospital length-of-stay }\end{array}$ & $\begin{array}{l}\text { No diff } \\
\text { clinical variables } \\
1.7 \pm 0.5 \text { vs. } 2.3 \pm 0.6 \\
\text { days } 8.2 \pm 2.3 \text { vs. } 12.5 \\
\pm 1.8 \text { days }\end{array}$ & $\begin{array}{l}\text { H-LOS: } \rightarrow \\
\text { ICU-LOS: } \rightarrow \\
\text { IR: N/A }\end{array}$ \\
\hline
\end{tabular}

All comparisons are intervention vs. control. Arrows showing; no difference $\rightarrow$, improvement/reduction $\downarrow$.

(Continuous positive airway pressure, CPAP; Positive pressure ventilation, PPV; Acute respiratory failure, ARF; Acute cardiogenic pulmonary edema, ACPE; Chronic obstructive pulmonary disease, COPD; Intubation rate, IR; Mortality in-hospital, IHM; Oxygen saturation, SpO2; Respiration rate, RR; Clinical variables, CV; Not Available, N/A; Not Reported, N/R; Hospital Length Of Stay, H-LOS; Intensive Care Length Of Stay, ICU-LOS. 
Table 2 Clarity and quality of the included studies

\begin{tabular}{|c|c|c|c|c|c|c|c|c|c|c|c|c|}
\hline Study & $\begin{array}{l}\text { Cheskes } \\
\text { et al. } \\
\text { [16] }\end{array}$ & $\begin{array}{l}\text { Dib } \\
\text { et al. } \\
\text { [27] }\end{array}$ & $\begin{array}{l}\text { Ducros } \\
\text { et al. [30] }\end{array}$ & $\begin{array}{l}\text { Frontin } \\
\text { et al. } \\
\text { [28] }\end{array}$ & $\begin{array}{l}\text { Gardtman } \\
\text { et al. } \\
\text { [22] }\end{array}$ & $\begin{array}{l}\text { Garuti } \\
\text { et al. [25] }\end{array}$ & $\begin{array}{l}\text { Hubble } \\
\text { et al. [24] }\end{array}$ & $\begin{array}{l}\text { Thompson } \\
\text { et al. } \\
\text { [23] }\end{array}$ & $\begin{array}{l}\text { Craven } \\
\text { et al. [29] }\end{array}$ & $\begin{array}{l}\text { Roessler } \\
\text { et al. [20] }\end{array}$ & $\begin{array}{l}\text { Schmiedbauer } \\
\text { et al. } \\
\text { [21] }\end{array}$ & $\begin{array}{l}\text { Weitz } \\
\text { et al. } \\
\text { [26] }\end{array}$ \\
\hline $\begin{array}{l}1.1 \text { The study } \\
\text { addresses an appropriate } \\
\text { and clearly focused question }\end{array}$ & $\ldots .$. &.. & $\ldots . .$. & $\ldots$ & $\ldots$ & $\ldots$ & $\ldots .$. & $\ldots . .$. & $\ldots . .$. & $\ldots$ &.. & $\ldots$ \\
\hline $\begin{array}{l}1.2 \text { The assignment of subjects } \\
\text { to treatment groups randomised }\end{array}$ & NA & NA & $\ldots$ & $\ldots$ & NA & NA & NA & $\ldots$ & NA & $\ldots$. & $\ldots$ & $\cdots$ \\
\hline $\begin{array}{l}1.3 \text { An } \\
\text { adequate concealment } \\
\text { method is used }\end{array}$ & NA & NA & $\cdot$ & $\ldots$ & NA & NA & NA & $\ldots$ & NA & $\ldots$. & $\ldots$ & $\cdot$ \\
\hline $\begin{array}{l}\text { 1.4 Subjects and investigators } \\
\text { are kept 'blind' to treatment } \\
\text { allocation }\end{array}$ & NA & NA &.. & $\ldots$ & NA & NA & NA & $\ldots$ & NA & $\cdot$ & $\cdot$ & $\cdot$ \\
\hline $\begin{array}{l}1.5 \text { The treatment and control } \\
\text { groups were similar at the } \\
\text { start of the trial }\end{array}$ & $\cdots$ &.. & $\cdots$ & $\ldots$ & $\ldots$ &.. & .. & $\ldots$ & .• & $\ldots$ & $\ldots$ &.. \\
\hline $\begin{array}{l}1.6 \text { The only difference between } \\
\text { the groups is the treatment } \\
\text { under investigation }\end{array}$ & $\cdots$ &.. & $\cdots$ & $\ldots$ &.. &.. &.. & $\ldots$ & $\ldots$ & .• & $\ldots$ &.. \\
\hline $\begin{array}{l}\text { 1.7 All relevant outcomes } \\
\text { measured in a standard, } \\
\text { valid and reliable way }\end{array}$ & $\cdots$ & $\ldots$ & $\cdots$ & $\cdots$ &.. & $\cdots$ & $\ldots$ & $\ldots$. & $\cdots$ & $\ldots$ & $\ldots$ & $\cdots$ \\
\hline $\begin{array}{l}1.8 \text { What percentage of the } \\
\text { individuals or clusters recruited } \\
\text { into each treatment arm of the } \\
\text { study dropped out before the } \\
\text { study was completed? }\end{array}$ & 00 & $\begin{array}{l}4 / \\
14911 / \\
238\end{array}$ & $\begin{array}{l}11 / 10713 / \\
100\end{array}$ & $\begin{array}{l}0 / 622 / \\
62\end{array}$ & 00 & NRNR & $\begin{array}{l}10 / 12024 / \\
95\end{array}$ & $1 / 351 / 36$ & $\begin{array}{l}9 / 71 \text { in } \\
\text { total }\end{array}$ & $\begin{array}{l}2 / 51 \text { in } \\
\text { total }\end{array}$ & 1/18 0/18 & $\begin{array}{l}0 / 13 \\
0 / 10\end{array}$ \\
\hline $\begin{array}{l}1.9 \text { All the subjects are } \\
\text { analysed in the groups } \\
\text { to which they } \\
\text { were (randomly) allocated }\end{array}$ & $\cdots$ & NA & $\cdots$ & $\ldots$ & $\ldots$ & NA & $\cdots$ & $\ldots$. &.. & $\ldots$ & $\cdot$ & $\cdots$ \\
\hline $\begin{array}{l}1.10 \text { Where the study } \\
\text { is carried out at more }\end{array}$ & NA &.. & $\ldots$ & NA & NA & NA & .. & NA & NA & NA & NA & NA \\
\hline
\end{tabular}


Table $\mathbf{2}$ Clarity and quality of the included studies (Continued)

than one site, results

are comparable for all sites

2.1 How well was the

study done to minimise bias?

2.2 Taking into account

clinical considerations,

your evaluation of the

methodolgy used, and

the statistical power of

the study, are you certain

that the overall effect i

$s$ due to the study intervention?

2.3 Are the results of this study directly applicable to

the patient group targeted by this review?

Well covered ....

Adequately addressed ...

Poorly addressed $\cdot \cdot$

Not addressed .

Not applicable NA

Not reported NR

Few/no criteria fulfilled -

Some criteria fulfilled +

All/most criteria fulfilled ++ 


\section{CPAP}

In the eight studies comparing supplemental CPAP to standard medical treatment alone, the number of patients in the intervention groups ranges between 35 and $214[16,22-25,27,28,30]$. Three studies are randomized controlled trials $[23,28,30]$. One study is prospective but not randomized [24]. Three studies are before and after studies $[16,22,25]$. One study is a cross-sectional study, where outcomes of patients receiving CPAP are compared to outcomes from those not receiving this intervention [27]. In three studies comparing supplemental CPAP to standard medical treatment alone, a reduction in mortality is found [23-25]. No studies show a significant increase in mortality with administration of CPAP. One study shows a lower H-LOS [25] and another study reports no difference in H-LOS [22]. Three studies report no difference in ICU-LOS [23,28,30]. Intubation rate is reduced with supplemental CPAP treatment in three studies [23,24,27]. According to our evaluation of clarity and quality of the studies, four studies either lack power to conclude on our outcomes of interest [25] or entail high risk of bias $[22,24,25,27]$ and were excluded from contribution to our conclusion. Four studies have acceptable power and risk of bias $[16,23,28,30]$. Of these, one smaller randomized controlled trial shows a reduction in both mortality and intubation rate [23]. The remaining three - one large descriptive study and two randomized and controlled studies show no effect on any of our outcomes of interest $[16,28,30]$, but there is a trend toward lower intubation rate with supplementary CPAP in two of these studies $[28,30]$. The prehospital, inhospital, and overall intubation rates are shown in Table 3.

\section{NIV}

In the four studies comparing supplemental prehospital NIV to standard medical treatment alone, the number of patients in the intervention groups ranges between 10 and $37[20,21,26,29]$. Three of the studies have a randomized and controlled design $[20,21,26]$. The fourth study is a prospective controlled study with five rescue units administering bi-level positive airway pressure as intervention group and five units administering standard medical treatment alone as control group [29]. There was no difference in mortality with supplemental NIV compared to standard medical treatment alone. In two studies, a reduction in ICU-LOS is found [20,21]. One study reports no difference in ICU-LOS [26]. No difference in total H-LOS is found in any of the studies. No difference in intubation rate is shown in any of the studies. According to our evaluation of clarity and quality of evidence, all four studies lack power to make conclusions on our outcomes of interest $[20,21,26,29]$ and two studies entail high risk of bias $[26,29]$. In two small studies with low risk of bias, a reduction in ICU-LOS and a trend toward decreased intubation rate with supplemental prehospital NIV is seen [20,21], see Table 3.

\section{Discussion}

Our principal findings are: 1) One of four studies of acceptable quality shows a lower mortality and intubation rate with supplemental prehospital CPAP compared to standard medical treatment alone, and the remaining three are neutral. A trend toward lower intubation rate with supplemental prehospital CPAP is seen in two studies. 2) There is insufficient evidence to conclude on the use of supplemental prehospital NIV.

\section{CPAP}

All the included studies are relatively small, and many lack the power to investigate hard endpoints like mortality. Three studies did indeed find a reduced mortality [23-25], but two studies had problems with the study design $[24,25]$. The risk of type two statistical errors in the included studies is high.

In the study by Cheskes et al. a trend towards increased mortality in the subgroups chronic heart failure, COPD and pulmonary edema was seen, but this was not found in the other studies and their result was not statistically significant [16].

Prehospital CPAP given as a supplement to standard medical treatment improves clinical endpoints like respiratory rate and arterial saturation [24,25,27], when compared to standard medical treatment alone. In studies where arterial gases were taken, an improvement was seen $[25,30] . \mathrm{PaO}_{2}$ improved and $\mathrm{pH}$ was higher in the intervention group in one study [25]. The other study showed lower $\mathrm{PCO}_{2}$ and normalization of $\mathrm{pH}$, but did not report on $\mathrm{PaO}_{2}$ as an endpoint [30]. These findings, combined with the lower intubation rate [23] and trend toward lower intubation rates $[28,30]$, indicate that CPAP may reduce prehospital intubation rates, but this needs to be verified. Whether or not this is beneficial, cannot be answered based on the current evidence.

Of the four studies with acceptable quality, two included patients with acute respiratory failure of any cause [16,23] and two included patients with ACPE $[28,30]$. The low number of studies does not allow us to conclude on differences between the conditions being treated for.

\section{NIV}

There is insufficient evidence to conclude on the effect of supplemental prehospital NIV compared to standard medical treatment alone. The failure to demonstrate differences in mortality, intubation rate, and H-LOS could be caused by type two errors in these small studies. Thus this does not mean that the strategy should be abandoned in future research. Studies in patients with COPD indicate that CPAP decreases inspiratory work of breathing [31]. 
Table 3 Intubation rates with supplemental prehospital CPAP/NIV compared to standard medical treatment alone

\begin{tabular}{|c|c|c|}
\hline Study & Supplemental prehospital CPAP/NIV & Standard medical treatment alone \\
\hline \multicolumn{3}{|l|}{ CPAP } \\
\hline Cheskes et al. 2013 & $31 / 214$ & $30 / 228$ \\
\hline Prehospital & $0 / 124$ & $1 / 228$ \\
\hline Inhospital & $31 / 214$ & $29 / 228$ \\
\hline Ducros et al. 2011 & $3 / 107$ & $6 / 100$ \\
\hline Prehospital & NA & NA \\
\hline Inhospital & NA & NA \\
\hline Frontin et al. 2011 & $2 / 60$ & $3 / 62$ \\
\hline Prehospital & $0 / 60$ & $1 / 62$ \\
\hline Inhospital & $2 / 60$ & $2 / 62$ \\
\hline Thompson et al. 2008 & $7 / 35$ & $17 / 34^{*}$ \\
\hline Prehospital & $0 / 35$ & $9 / 34$ \\
\hline Inhospital & $7 / 35$ & $8 / 34$ \\
\hline Dib et al. 2012 & NA & NA \\
\hline Prehospital & $4 / 149$ & $11 / 238^{*}$ \\
\hline Inhospital & NA & NA \\
\hline Gardtman et al. 2000 & NA & NA \\
\hline Prehospital & NA & NA \\
\hline Inhospital & NA & NA \\
\hline Garuti et al. 2010 & $0 / 35$ & $14 / 125$ \\
\hline Prehospital & $0 / 35$ & $14 / 125$ \\
\hline Inhospital & $0 / 35$ & $0 / 125$ \\
\hline Hubble et al 2006 & $10 / 120$ & $24 / 95^{*}$ \\
\hline Prehospital & $5 / 120$ & $7 / 95$ \\
\hline Inhospital & $5 / 120$ & $17 / 95$ \\
\hline \multicolumn{3}{|l|}{ NIV } \\
\hline Craven et al 2000 & $4 / 37$ & $7 / 25$ \\
\hline Prehospital & $0 / 37$ & $6 / 25$ \\
\hline Inhospital & $4 / 37$ & $1 / 25$ \\
\hline Roessler et al 2011 & $1 / 24$ & $6 / 25$ \\
\hline Prehospital & $0 / 24$ & $1 / 25$ \\
\hline Inhospital & $1 / 14$ & $5 / 25$ \\
\hline Schmidbauer et al 2011 & $3 / 18$ & $7 / 18$ \\
\hline Prehospital & $1 / 18$ & $0 / 18$ \\
\hline Inhospital & $2 / 18$ & $0 / 18$ \\
\hline Weitz et al 2007 & NA & NA \\
\hline Prehospital & NA & NA \\
\hline Inhospital & NA & NA \\
\hline
\end{tabular}

*=statistical significant difference. Studies rated as having acceptable size and quality are bold.

The addition of pressure support ventilation to positive end expiratory pressure, increases tidal volume in proportion to the amount of pressure applied and theoretically relives inspiratory muscles [32]. Thus theoretically, NIV should be advantageous and in all of the included studies measuring these, vital signs like respiratory rate improve when NIV is used. Arterial saturation significantly improves with supplemental NIV compared to standard medical treatment alone, including high fractions of inspired oxygen, in three of the studies included in this review $[20,26,29]$. In the fourth study, improvement in arterial saturation was more pronounced in the intervention group, 
but failed to reach level of significance [21]. We speculate that these improvements in vital signs may lead to a better patient outcome, but this was not demonstrated in the included studies - most likely because of low sample sizes. In both studies with acceptable risk of bias but low sample size, a trend toward lower intubation rate with supplementary NIV, compared to standard medical treatment alone, is found $[20,21]$. However, the small sample sizes in these studies prohibit us from making solid conclusions on the use of prehospital supplemental NIV.

\section{General considerations}

The equipment used to administer CPAP or NIV includes an external pressure regulator (WhisperFlow [23,24,27,30]), a turbulent flow valve (Boussignac [28]), helmet CPAP (Castar-Starmed, Flow-meter [25]), a ventilatory system (Respironics 330000 [29]) and a portable ventilator (Oxylog $3000[20,21,26])$. The last two devices are used to administer NIV, and this equipment is technically more sophisticated than the equipment used to deliver CPAP. The medical staffing of the dispatched rescue teams in the included studies was heterogeneous. Physicians administered CPAP/NIV in five studies [20,21,26,28,30] and paramedics or emergency medical technicians administered CPAP/NIV in another five studies $[16,23,24,27,29]$. Nurses administered CPAP in one study [25] and in one study both paramedics and, for $25 \%$ of the time, nurses provided treatment with CPAP [22]. Further comparison and analysis of the equipment used, is beyond the scope of this review.

None of the studies included in this review report problems with safety, or with easy of use, when administrating CPAP or NIV, regardless of the treating clinicians' qualifications. Only physicians provided NIV with the Oxylog 3000. The low number of included studies, and their varying study design, does not allow us to distinguish between conditions being treated for or to compare those who administered CPAP.

A recent systematic review and meta-analysis of randomized controlled trials on prehospital CPAP/NIV by $\mathrm{Mal}$ et al. finds a reduction in the need for inhospital invasive ventilation and mortality, with the use of prehospital noninvasive positive pressure ventilation [33]. The review by $\mathrm{Mal}$ et al. includes only randomized and controlled studies. In their review, seven heterogeneous studies on both CPAP and NIV, with a total of 632 patients, are combined in a meta-analysis. They also included one study that was excluded from our review [18]. This study by Plaisance et al. compares different treatment algorithms, both involving CPAP and medical treatment, at different time intervals. A large proportion of the studies included in the meta-analysis by Mal et al. were relatively small. This can increase the risk of overestimating the effect of the intervention due to publication bias, as small studies with negative findings are less likely to be accepted for publication. A recent review by Simpson et al. finds that prehospital CPAP/NIV appears to be safe and feasible therapy that results in faster improvement in physiological status, and that it may decrease the need for intubation, when compared to delayed administration in the emergency department [34]. They state there is weak evidence that NIV may decrease mortality, which is not in agreement with our findings. In their review, the majority of articles included are noncomparative descriptive studies only on ACPE, and they included three studies that were excluded from our review [17-19]. Simpson et al. recognized, but did not discriminate between different forms of NIV.

\section{Limitations}

The risk of publication bias is, as for all reviews, a weakness of this study. No studies in non-English languages met our inclusion criteria, but among our excluded studies were studies published in German, Spanish, Japanese, French, and Russian. However, there is a risk that studies published in other languages than English to a lesser extend are indexed in the searched databases. This can theoretically produce an overestimation of the positive effects of CPAP or NIV [35].

We used the SIGN 50 checklist designed for randomized controlled trials, because this checklist is relevant when considering nonrandomized studies as well. These study evaluations inevitably involve a degree of subjective judgment.

The external validity of our review is difficult to outline as the included studies are from different parts of the world, with different geographic characteristics, different medical staffing, and with different structure of the emergency medical services. Standard medical treatment used in the included studies cannot be regarded as uniform, although medical treatment of acute exacerbations of COPD and ACPE is well established. This could make the results less comparable.

When considering all patients attended, few are intubated in the prehospital setting [12,36,37]. In the Scandinavian countries, as in the majority in Europe, physicians provide prehospital advanced airway management and have the ability to intubate the trachea on scene. The treating clinicians in the included studies are often paramedics and not experienced physicians - this may have influenced intubation rates [38].

The pressures applied when administrating CPAP or NIV varied among the included studies. Reported pressure settings when administrating CPAP in the included trials, ranged from 5 to $10 \mathrm{~cm} \mathrm{H}_{2} \mathrm{O}$. One study examining NIV adjusted pressures according to a predefined protocol, and up to $20 \mathrm{~cm} \mathrm{H}_{2} \mathrm{O}$ support pressure was given [20]. Four studies did not report on pressure settings $[16,21,22,29]$. Different pressure levels could be clinically relevant when comparing interventions. 


\section{Unanswered questions and future research}

Supplemental prehospital CPAP seems to improve vital signs, compared to standard medical treatment alone, and there is a trend toward lower intubation rate. This needs to be confirmed in larger, randomized controlled trials, and whether it is beneficial in terms of lower mortality or morbidity also needs clarification.

Supplemental prehospital NIV, compared to standard medical treatment alone, also seems to improve vital signs, but it is unknown whether this affects patient outcome. With regards to what we know from the inhospital setting, it seems reasonable to include patients with acute exacerbations of COPD in future studies [6]. The application of NIV in the prehospital setting, and thereby early initiation, may be more advantageous in case of long distances to the receiving hospitals; this could also be a focus for future research. Interestingly, there were only few reported problems with mask-tolerance in studies included in this review - this is a well-described problem inhospital, and it is unlikely that these issues are smaller in the prehospital setting even if the treatment time is short. This subject and the patients' perception of mask treatment, in the prehospital setting, could also be a focus for future research $[39,40]$.

\section{Conclusion}

The current evidence shows no difference in mortality or hospital length of stay, but a trend toward reduced intubation rate with prehospital supplemental CPAP compared to standard medical treatment alone. This needs to be verified in larger, randomized controlled trials. The current evidence regarding prehospital supplemental NIV is scarce, and the conducted studies are too small to make reasonable conclusions, but justify further research.

\section{Competing interests}

The authors declare that they have no competing interest.

\section{Authors' contributions}

SAB and MTB (both investigators) designed the study, carried out the searches and extracted study details from the included articles. SAB and ISR evaluated the quality of the studies. ISR designed tables and figures. HK and EFC participated in designing the study and revising the manuscript along with SAB, MTB, and ISR. All authors have approved of the final manuscript.

\section{Author details}

${ }^{1}$ Department of Anesthesiology, Hospital of Southern Jutland, Southern Jutland, Denmark. ${ }^{2}$ Prehospital Research Department, Prehospital Emergency Medical Services, Central Denmark Region, Denmark. ${ }^{3}$ Research Center for Emergency Medicine, Aarhus University Hospital, Aarhus, Denmark.

Received: 25 April 2014 Accepted: 5 November 2014

Published online: 22 November 2014

\section{References}

1. Stiell IG, Spaite DW, Field B, Nesbitt LP, Munkley D, Maloney J, Dreyer J, Toohey LL, Campeau T, Dagnone E, Lyver M, Wells GA: Advanced life support for out-of-hospital respiratory distress. N Engl J Med 2007, 356:2156-2164
2. Fevang E, Lockey D, Thompson J, Lossius HM, Torpo Research C: The top five research priorities in physician-provided pre-hospital critical care: a consensus report from a European research collaboration. Scand J Trauma Resusc Emerg Med 2011, 19:57.

3. Hess DR: Noninvasive ventilation for acute respiratory failure. Respir Care 2013, 58:950-972.

4. Mehta S, Hill NS: Noninvasive ventilation. Am J Respir Crit Care Med 2001, 163:540-577.

5. Vital FM, Ladeira MT, Atallah AN: Non-invasive positive pressure ventilation (CPAP or bilevel NPPV) for cardiogenic pulmonary oedema. Cochrane Database Syst Rev 2013, 5:CD005351.

6. Ram FS, Picot J, Lightowler J, Wedzicha JA: Non-invasive positive pressure ventilation for treatment of respiratory failure due to exacerbations of chronic obstructive pulmonary disease. Cochrane Database Syst Rev 2004, 3:CD004104.

7. Nourdine K, Combes P, Carton MJ, Beuret P, Cannamela A, Ducreux JC: Does noninvasive ventilation reduce the ICU nosocomial infection risk? A prospective clinical survey. Intensive Care Med 1999, 25:567-573.

8. Girou E, Schortgen F, Delclaux C, Brun-Buisson C, Blot F, Lefort Y, Lemaire F, Brochard $L$ : Association of noninvasive ventilation with nosocomial infections and survival in critically ill patients. JAMA 2000, 284:2361-2367.

9. Guerin C, Girard R, Chemorin C, De Varax R, Fournier G: Facial mask noninvasive mechanical ventilation reduces the incidence of nosocomial pneumonia. A prospective epidemiological survey from a single ICU. Intensive Care Med 1997, 23:1024-1032.

10. Peters J, van Wageningen B, Hendriks I, Eijk R, Edwards M, Hoogerwerf N, Biert J: First-pass intubation success rate during rapid sequence induction of prehospital anaesthesia by physicians versus paramedics. Eur J Emerg Med 2014, [Epub ahead of print].

11. Ufberg JW, Bushra JS, Karras DJ, Satz WA, Kueppers F: Aspiration of gastric contents: association with prehospital intubation. Am J Emerg Med 2005, 23:379-382.

12. Rognas L, Hansen TM, Kirkegaard H, Tonnesen E: Pre-hospital advanced airway management by experienced anaesthesiologists: a prospective descriptive study. Scand J Trauma Resusc Emerg Med 2013, 21:58.

13. Celikel T, Sungur M, Ceyhan B, Karakurt S: Comparison of noninvasive positive pressure ventilation with standard medical therapy in hypercapnic acute respiratory failure. Chest 1998, 114:1636-1642.

14. Plant PK, Owen JL, Elliott MW: Early use of non-invasive ventilation for acute exacerbations of chronic obstructive pulmonary disease on general respiratory wards: a multicentre randomised controlled trial. Lancet 2000, 355:1931-1935.

15. Scottish Intercollegiate Guidelines Network: SIGN 50: a Guideline Developer`s Handbook. Scotland: Scottish Intercollegiate Guidelines Network, Healthcare Improvement; 2008. http://www.sign.ac.uk/ methodology/checklists.html.

16. Cheskes S, Turner L, Thomson S, Aljerian N: Does prehospital continuous positive airway pressure impact the rate of intubation and mortality of acute respiratory emergencies? Can J Emerg Med 2013, 15:S52.

17. Foti G, Sangalli F, Berra L, Sironi S, Cazzaniga M, Rossi GP, Bellani G, Pesenti A: Is helmet CPAP first line pre-hospital treatment of presumed severe acute pulmonary edema? Intensive Care Med 2009, 35:656-662.

18. Plaisance P, Pirracchio R, Berton C, Vicaut E, Payen D: A randomized study of out-of-hospital continuous positive airway pressure for acute cardiogenic pulmonary oedema: physiological and clinical effects. Eur Heart J 2007, 28:2895-2901.

19. Hubble MW, Richards ME, Wilfong DA: Estimates of cost-effectiveness of prehospital continuous positive airway pressure in the management of acute pulmonary edema. Prehosp Emerg Care 2008, 12:277-285.

20. Roessler MS, Schmid DS, Michels P, Schmid O, Jung K, Stober J, Neumann P, Quintel M, Moerer O: Early out-of-hospital non-invasive ventilation is superior to standard medical treatment in patients with acute respiratory failure: a pilot study. Emerg Med J 2012, 29:409-414.

21. Schmidbauer W, Ahlers O, Spies C, Dreyer A, Mager G, Kerner T: Early prehospital use of non-invasive ventilation improves acute respiratory failure in acute exacerbation of chronic obstructive pulmonary disease. Emerg Med J 2011, 28:626-627.

22. Gardtman M, Waagstein L, Karlsson T, Herlitz J: Has an intensified treatment in the ambulance of patients with acute severe left heart failure improved the outcome? Eur J Emerg Med 2000, 7:15-24. 
23. Thompson J, Petrie DA, Ackroyd-Stolarz S, Bardua DJ: Out-of-hospital continuous positive airway pressure ventilation versus usual care in acute respiratory failure: a randomized controlled trial. Ann Emerg Med 2008, 52:232-241. 241 e231.

24. Hubble MW, Richards ME, Jarvis R, Millikan T, Young D: Effectiveness of prehospital continuous positive airway pressure in the management of acute pulmonary edema. Prehosp Emerg Care 2006, 10:430-439.

25. Garuti G, Bandiera G, Cattaruzza MS, Gelati L, Osborn JF, Toscani S, Confalonieri M, Lusuardi M: Out-of-hospital helmet CPAP in acute respiratory failure reduces mortality: a study led by nurses. Monaldi Arch Chest Dis 2010, 73:145-151.

26. Weitz G, Struck J, Zonak A, Balnus S, Perras B, Dodt C: Prehospital noninvasive pressure support ventilation for acute cardiogenic pulmonary edema. Eur J Emerg Med 2007, 14:276-279.

27. Dib JE, Matin SA, Luckert A: Prehospital use of continuous positive airway pressure for acute severe congestive heart failure. J Emerg Med 2012, 42:553-558.

28. Frontin P, Bounes V, Houze-Cerfon CH, Charpentier S, Houze-Cerfon V, Ducasse JL: Continuous positive airway pressure for cardiogenic pulmonary edema: a randomized study. Am J Emerg Med 2011, 29:775-781.

29. Craven RA, Singletary N, Bosken L, Sewell E, Payne M, Lipsey R: Use of bilevel positive airway pressure in out-of-hospital patients. Acad Emerg Med 2000, 7:1065-1068.

30. Ducros L, Logeart D, Vicaut E, Henry P, Plaisance P, Collet JP, Broche C, Gueye P, Vergne M, Goetgheber D, Pennec PY, Belpomme V, Tartiere JM, Lagarde S, Placente M, Fievet ML, Montalescot G, Payen D: CPAP for acute cardiogenic pulmonary oedema from out-of-hospital to cardiac intensive care unit: a randomised multicentre study. Intensive Care Med 2011, 37:1501-1509.

31. Appendini L, Patessio A, Zanaboni S, Carone M, Gukov B, Donner CF, Rossi $A$ : Physiologic effects of positive end-expiratory pressure and mask pressure support during exacerbations of chronic obstructive pulmonary disease. Am J Respir Crit Care Med 1994, 149:1069-1076.

32. Meyer TJ, Hill NS: Noninvasive positive pressure ventilation to treat respiratory failure. Ann Intern Med 1994, 120:760-770.

33. Mal S, McLeod S, lansavichene A, Dukelow A, Lewell M: Effect of Out-ofHospital Noninvasive Positive-Pressure Support Ventilation in Adult Patients With Severe Respiratory Distress: A Systematic Review and Meta-analysis. Ann Emerg Med 2013, 63:600-607.

34. Simpson PM, Bendall JC: Prehospital non-invasive ventilation for acute cardiogenic pulmonary oedema: an evidence-based review. Emerg Med J 2011, 28:609-612.

35. Egger M, Smith GD: Bias in location and selection of studies. BMJ 1998, 316:61-66.

36. McQueen C, Crombie N, Hulme J, Cormack S, Hussain N, Ludwig F, Wheaton S: Prehospital anaesthesia performed by physician/critical care paramedic teams in a major trauma network in the UK: a 12 month review of practice. Emerg Med J 2013, 0:1-5

37. Helm M, Hossfeld B, Schafer S, Hoitz J, Lampl L: Factors influencing emergency intubation in the pre-hospital setting-a multicentre study in the German Helicopter Emergency Medical Service. Br J Anaesth 2006, 96:67-71.

38. Garner A, Rashford S, Lee A, Bartolacci R: Addition of physicians to paramedic helicopter services decreases blunt trauma mortality. Aust N Z J Surg 1999, 69:697-701.

39. Chacur FH, Vilella Felipe LM, Fernandes CG, Lazzarini LC: The total face mask is more comfortable than the oronasal mask in noninvasive ventilation but is not associated with improved outcome. Respiration 2011, 82:426-430.

40. Navalesi P, Costa R, Ceriana P, Carlucci A, Prinianakis G, Antonelli M, Conti G, Nava S: Non-invasive ventilation in chronic obstructive pulmonary disease patients: helmet versus facial mask. Intensive Care Med 2007, 33:74-81.

\section{doi:10.1186/s13049-014-0069-8}

Cite this article as: Bakke et al: Continuous positive airway pressure and noninvasive ventilation in prehospital treatment of patients with acute respiratory failure: a systematic review of controlled studies. Scandinavian Journal of Trauma, Resuscitation and Emergency Medicine $201422: 69$

\section{Submit your next manuscript to BioMed Central and take full advantage of:}

- Convenient online submission

- Thorough peer review

- No space constraints or color figure charges

- Immediate publication on acceptance

- Inclusion in PubMed, CAS, Scopus and Google Scholar

- Research which is freely available for redistribution 